\title{
Esophago-pulmonary fistula manifesting as recurrent pneumonias and migrating mediastinal calcifications
}

\author{
Jad Kebbe1, Sai Yendamuri2, Charles Roche3, Kassem Harris4, Samjot Singh Dhillon5 \\ 1 Department of Medicine, Division of Pulmonary, Critical Care and Sleep Medicine, University of Oklahoma \\ Health Sciences Center, Oklahoma City, OK \\ 2 Department of Thoracic Surgery, Roswell Park Cancer Institute, Buffalo, NY \\ 3 Department of Radiology, Roswell Park Cancer Institute, Buffalo, NY \\ 4 Department of Pulmonary and Critical Care Medicine, Section of Interventional Pulmonology Westchester \\ Medical Center, Valhalla, NY \\ 5 Department of Medicine, Division of Pulmonary Medicine and Interventional Pulmonology. Roswell Park \\ Cancer Institute, Buffalo, NY, USA
}

\begin{abstract}
A 43-year old man presented with recurrent pneumonias, episodes of hemoptysis and an enlarging right lower lobe mass. A clear diagnosis was not previously established in spite of multiple radiological evaluations and biopsies. Meticulous review of his CT imaging showed that he had subcarinal calcification on his prior CT scans, which had decreased in size and now multiple new small areas of calcifications were seen in the right lower lobe lesion. An esophago-pulmonary fistula due to migration of mediastinal calcifications was suspected which was identified on careful review of the CT chest and confirmed by esophagogastroduodenoscopy. Patient had surgical repair with complete recovery.
\end{abstract}

Corresponding author: Samjot Singh Dhillon, Department of Medicine, Section of Pulmonary Medicine, Roswell Park Cancer Institute, Elm and Carlton Street, Buffalo, NY 14263, USA.

Tel. +1.716.845-1487 - Fax +1.716.845-8346.

E-mail: Samjot.dhillon@roswellpark.org

Key words: Esophago-pulmonary fistula; broncholith; mediastinal calcification; pneumonia; hemoptysis.

Conflict of interest: the authors declare no potential conflict of interest.

Authors contributions: JK, SSD, wrote the manuscript; SY, CR, KH contributed to review, inclusion of data respective to their specialties and their contribution to the case. All authors reviewed the manuscript and approved it.

Received for publication: 28 January 2017

Accepted for publication: 19 March 2017

CC Copyright J. Kebbe et al., 2017

Tipografia PI-ME Editrice, Italy

Monaldi Archives for Chest Disease 2017; 87:813

doi: $10.4081 /$ monaldi.2017.813

This article is distributed under the terms of the Creative Commons Attribution Noncommercial License (by-nc 4.0) which permits any noncommercial use, distribution, and reproduction in any medium, provided the original author(s) and source are credited.

\section{Introduction}

Esophago-pulmonary fistula (EPF) is an uncommon condition that can be congenital or acquired after surgery, corrosive ingestions, malignancy or infections. Presentation is varied and includes chronic cough, recurrent infections and massive hemoptysis and clinical picture may mimic various infection and inflammatory conditions resulting in significant delays in diagnosis. Surgical treatment is preferred, yet endoscopic stent placement is an option for medically marginal patients. We report the unusual clinical presentation of esophago-pulmonary fistula which remained undiagnosed for years in a man with recurrent pneumonia, waxing and waning right lower lobe (RLL) infiltrates and migratory calcifications in the mediastinum and RLL. Thorough history and careful review of sequential radiographic examination were key for accurate diagnosis and definitive treatment.

\section{Case Report}

A 43-year-old man, ex-smoker of 2 packs per day, presented for an evaluation of an abnormal computed tomogram (CT) of the chest. He was exposed to solvents, but denied alcohol abuse. His only medications were inhaled corticosteroids with a long-acting beta agonist for treatment of mild chronic obstructive pulmonary disease (COPD). He was exasperated following visits to various hospitals and physicians over the preceding years, during which the possibility of lung cancer was repeatedly entertained, yet no conclusive diagnosis had been established.

About three years earlier, he had developed cough, weight loss, night sweats, exertional dyspnea, and was treated for pneumonia. Chest CT was interpreted as showing a RLL mass, emphysema, and calcifications in the subcarinal and right hilar regions (Figure 1). Sputum acid-fast smears, cultures, and a purified protein derivative skin test for tuberculosis were negative. He did not have any history of distant travel or exposure to tuberculosis. A bronchoscopy was reported as non-diagnostic with negative cultures for bacteria, fungi and acid-fast bacteria. Months later, the RLL lesion increased in size (Figure 2). CT-guided transthoracic biopsy revealed inflammation without malignancy. Since he was feeling better and gaining weight, he declined further invasive procedures. A follow-up chest CT reported a decrease in size of the lesion (Figure 3). 


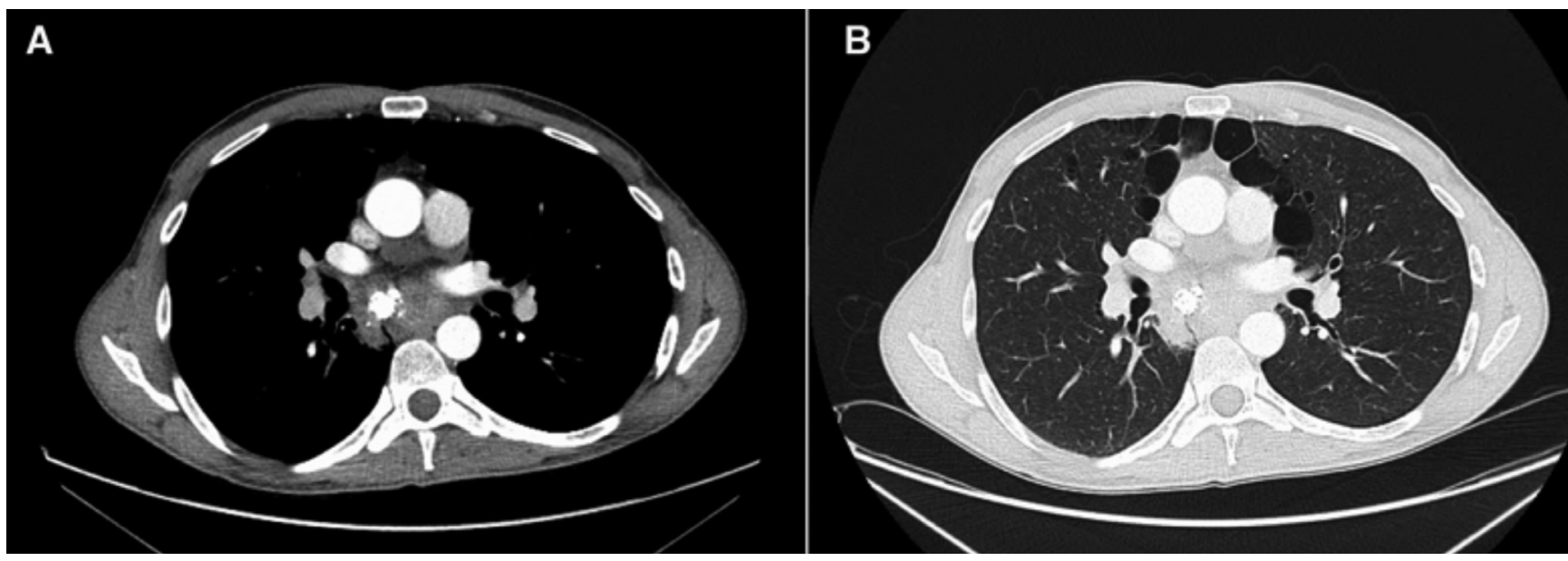

Figure 1. Axial CT chest with mediastinal (A) and lung (B) windows showing emphysema, and subcarinal calcification and right lower lobe infiltrate.

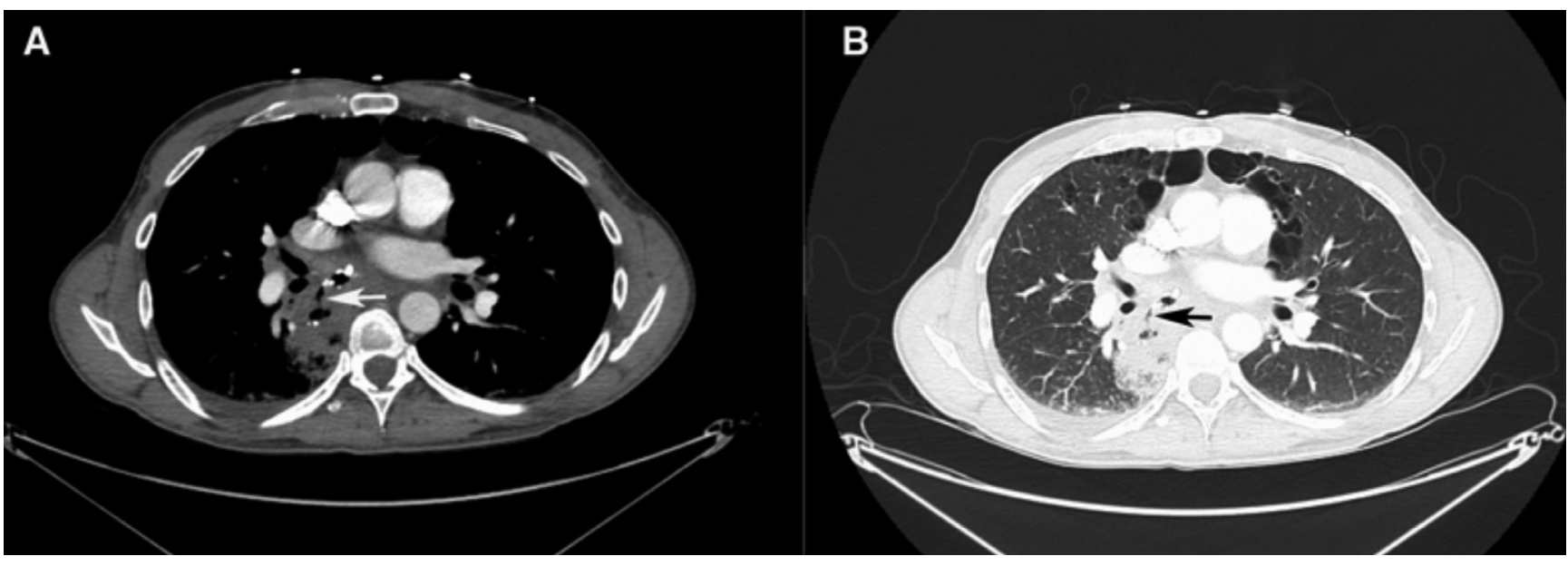

Figure 2. A,B) CT chest showing increase in right lower lobe infiltrate with scattered small calcifications. Careful review of this old CT showed that a fistulous track (white and black arrow) was present even at that time.

Over the following 2 years, episodes of dyspnea and low-grade fevers caused multiple visits to various hospitals and providers. Antibiotic courses coupled with corticosteroids led to some improvement. Nonetheless, chest pain and mild hemoptysis developed several weeks prior to this presentation. A chest CT angiogram revealed no pulmonary embolus, but the RLL opacity had again increased in size and parenchymal calcifications were noted (Figure 4). He was thus referred to us for evaluation of possible lung cancer. Physical examination merely noted dry right basilar rales. The cough was not associated with eating food or drinking liquids.

The persistence of a RLL infiltrate with a varying degree of consolidation led to a consideration of structural or congenital abnormalities, immune deficiency and autoimmune disorders in the differential. His recent CT imaging (Figure 4) provided a vital clue as it showed significantly less subcarinal calcification than the initial CT, but instead multiple new small parenchymal calcifications were noted. The possibility of migrating mediastinal calcification resulting with recurrent mediastinitis with esophagus-to-pulmonary parenchymal fistula formation was entertained. Hence, a thin section chest CT with barium esophagram was performed, and other prior CT scans were obtained from other institutions. There was no barium leakage into the airway, but a small fistulous tract was identified from the right lateral wall of the mid esophagus, extending into the pulmonary parenchyma, and communicating with branches of the RLL bronchus (Figure 5).

Bronchoscopy demonstrated edema of the posterior opening of the RLL superior segment without endobronchial lesions. Esophagogastroduodenoscopy revealed an $8 \mathrm{~mm}$ epithelialized opening along the right wall of the esophagus at $35 \mathrm{~cm}$ from the incisors, representing a fistula (Figure 6). There was no stricture, hence a stent was not placed as it was hypothesized that it would likely migrate. Moreover, the patient was deemed a good candidate for surgical repair. He was treated with amoxicillin/clavulanic acid and then underwent right posterolateral thoracotomy and successful proximal closure of the fistula with interposition of an intercostal muscle flap. Surgical resection of lung parenchyma was not performed as the lung parenchyma was felt to be salvageable by the thoracic surgery team. Histologically, the fistula was 


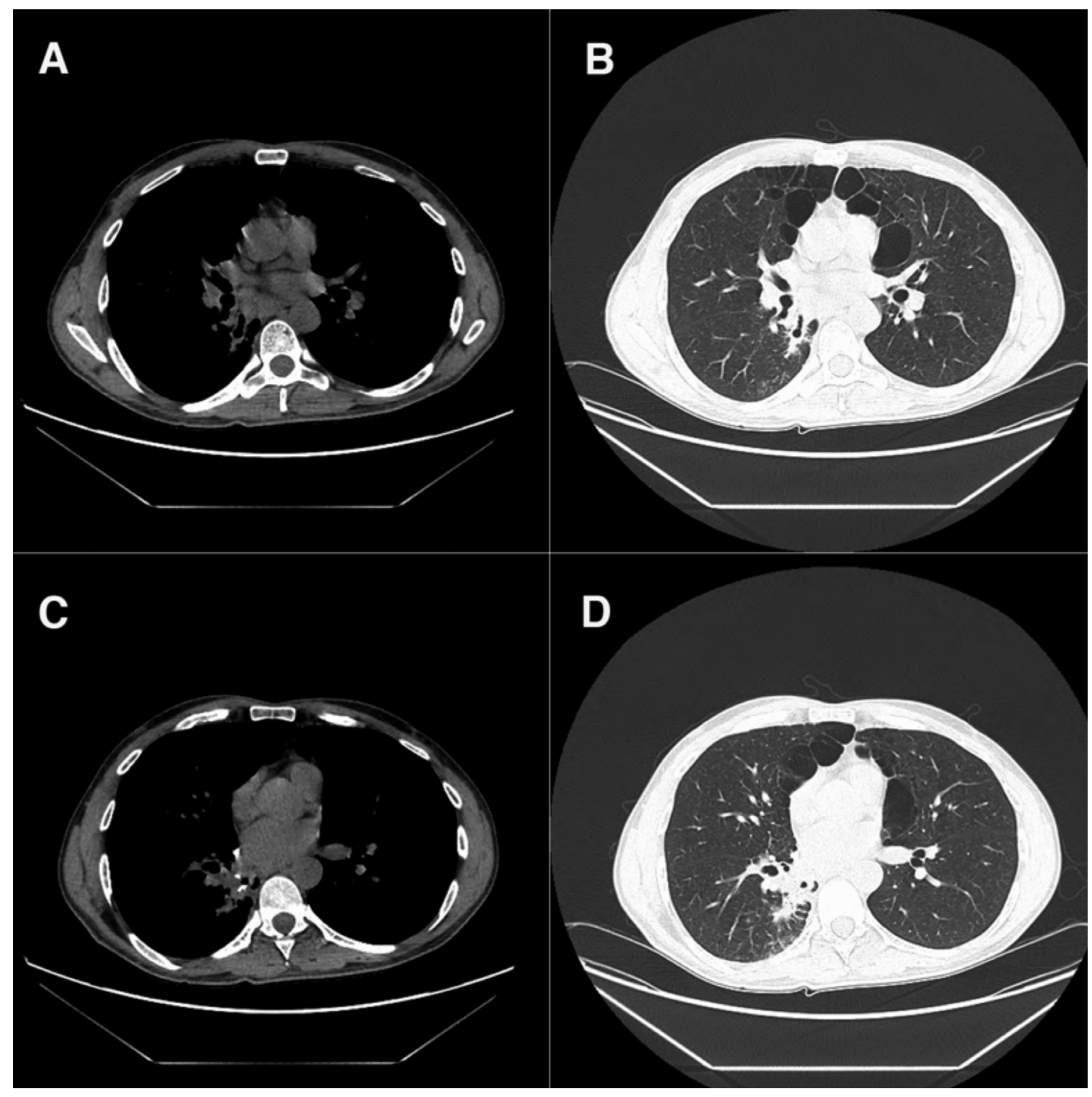

Figure 3. A-D) CT Chest with corresponding mediastinal and lung windows showing decrease in size of the right lower lobe infiltrate along with less subcarinal calcification and new multiple small foci of calcifications in the infiltrate.

lined by squamous epithelium, and exhibited no evidence of malignancy on pathology and tissue cultures were negative. Urine histoplasma antigen and serum serology for histoplasma and Coccidioides were negative. The mediastinal calcification was presumed to be due to old histoplasma infection as it is common infection in our geographical area and serology and cultures may not be helpful in cases with distant history of infection. Anti-fungal therapy was not indicated. The patient had an uneventful post-operative course, with removal of chest tube on post-operative day three and discharge on day five. The patient remains free of symptoms ten-months after surgery.

\section{Discussion}

EPF may be congenital or acquired [1,2]. It could be a complication of toxic ingestions or achalasia [3]. Fistula formation may also follow mediastinal infection (particularly granulomatous), malignancy, chemotherapy, trauma, thoracic surgery or lung transplantation [4-7]. Mediastinal lymph node calcifications are often the result of the healing process of an inflammatory condition affecting the mediastinum. Common etiologies are granulomatous infections 


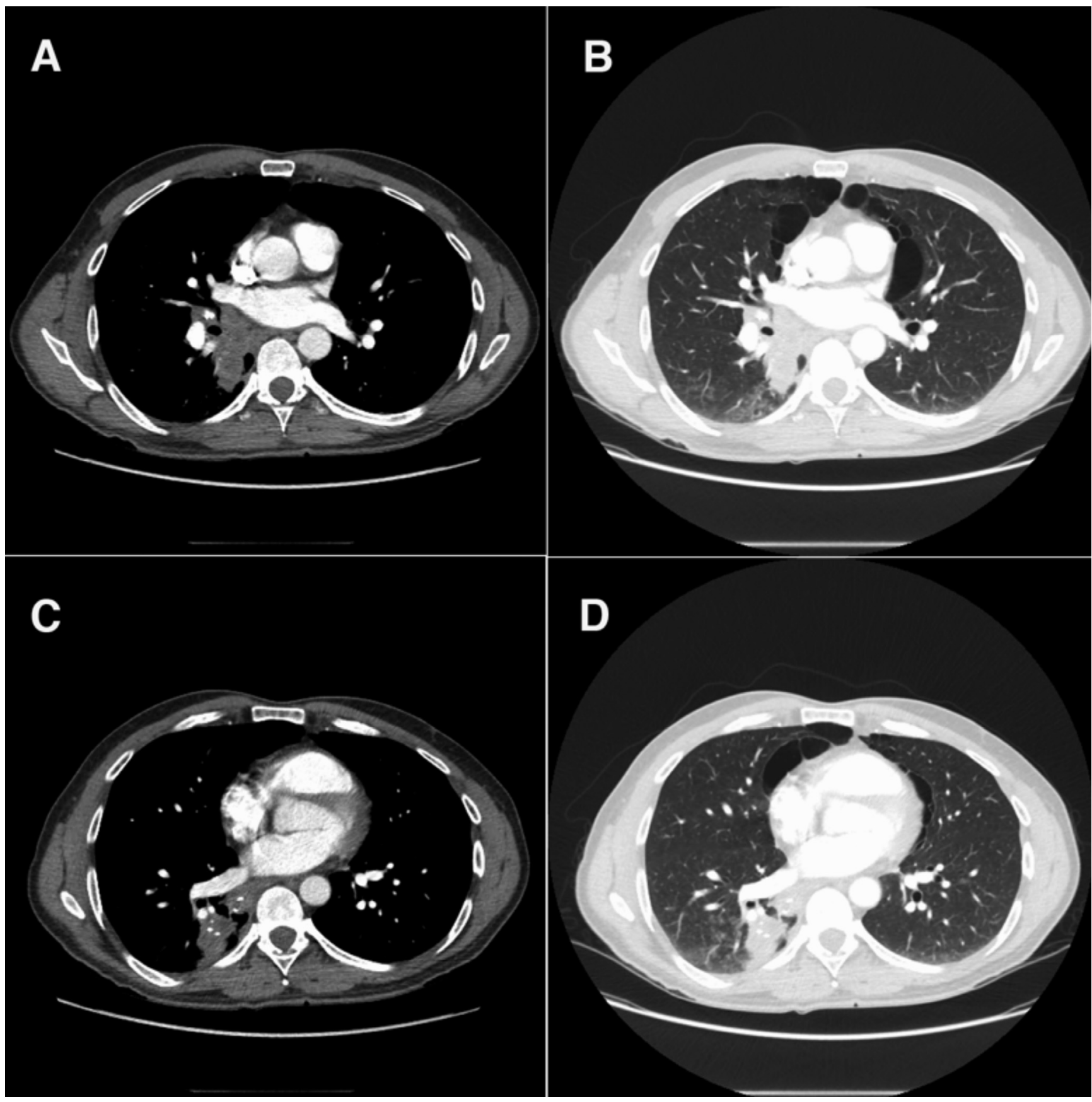

Figure 4. CT chest with corresponding mediastinal and lung windows showing increasing right lower lobe infiltrate with scattered parenchymal calcifications.

(Mycobacterium tuberculosis, Histoplasma capsulatum), silicosis and post-operative mediastinitis. Calcifications may erode into a bronchus. Resultant lithoptysis (expectoration of a foreign body in the form of a stone) has been described for centuries, dating back to Aristotle and Galen [8]. Consequences could be severe, ranging from chronic cough to exsanguination. Rarely, congenital fistulas may get diagnosed only in adulthood [1].

Although the esophagus has more proximity to the left main stem, EPF may have a predilection to the right side due to a higher number of lymph nodes in this area [9]. A case series reported common symptoms of intractable cough, typically worse after drinking, dysphagia and recurrent pneumonia [9]. Bronchiectasis and hemoptysis may occur [10]. Physical examination is generally underwhelming. Unlike in the reported series, our patient exhibited no cough with deglutition, yet had recurrent pneumonia and hemoptysis.

If the diagnosis of EPF is delayed, recurrent presentations could be frustrating. Chest imaging is usually abnormal: common findings are pulmonary infiltrates and calcified granulomas or lymph nodes. While esophagography has been historically the most used modality, a high index of clinical suspicion supported by radiographic findings may 


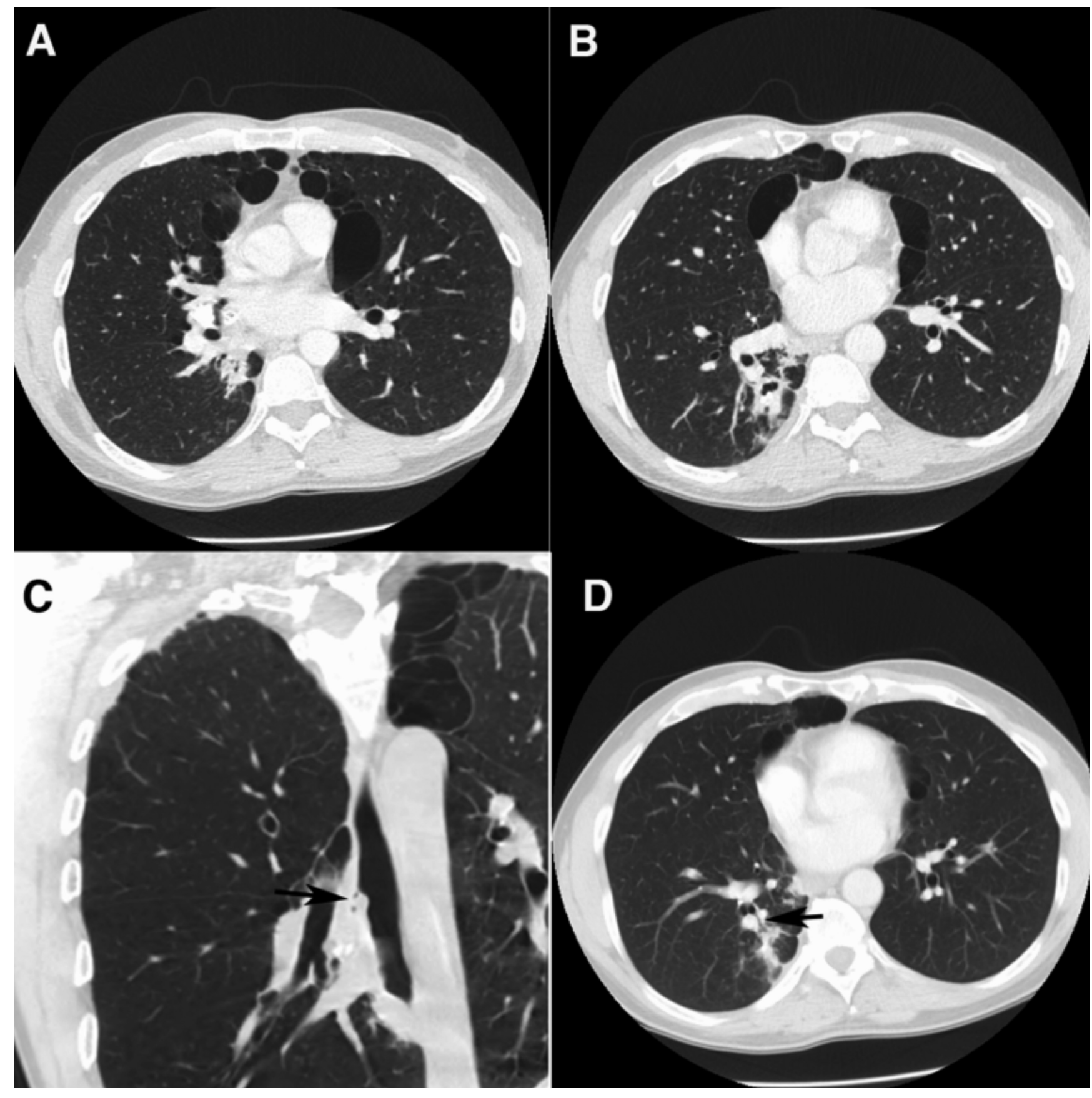

Figure 5. A,B) Axial CT chest demonstrating a decrease in size of right lower lobe infiltrate. Coronal CT (C) showing the fistula from esophagus to right lower lobe and axial image (D) showing fistula communicating with a right lower lobe basal segment (black arrows).

lead to diagnosis on chest tomography. Once identified, correction is warranted, whether endoscopically or surgically [11-13].

Endoscopically, stenting has been the most used modality [11]. It may be efficient when a stricture is present, thus achieving two therapeutic objectives of restoring the patency of the esophagus and holding the stent in place. Nonetheless, migration of the stent has been reported, and many a time, persistence or recurrence of symptoms. It remains the procedure of choice in those unsuitable for surgery. Conversely, surgical therapy is the treatment of choice in a medically fit patient. While at times not successful on the first attempt, and recurrence has been described, it often is a definitive therapy. In the largest retrospective review series [ 35 patients), single-stage primary repair of both airway and esophageal defects with tissue flap interposition was successful in most patients, with a low rate of complications and mortality [13].

Since delays in diagnosis of up to 6 years have been reported, with a mean of 21 months [9], attention to clues is paramount. Our patient likely had migration of mediastinal calcifications leading to perforation of the esophagus and erosion into the pulmonary parenchyma and bronchi thus, resulting in recurrent episodes of me- 


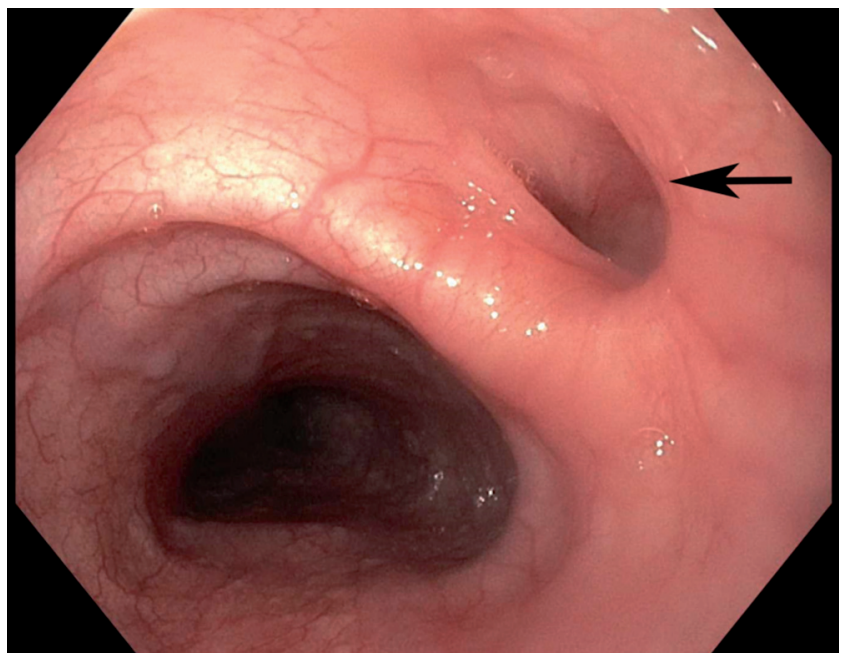

Figure 6. Esophagogastroduodenoscopy showing the small opening of the fistula (black arrow) without a stricture.

diastinitis and pneumonia. The migration may be due to slow and persistent local erosion of mediastinal soft-tissue by firm calcified broncholith facilitated by continuous motion of mediastinal tissue by respiratory motion and cardiac pulsations. Multiple courses of antibiotics and corticosteroids did not result in resolution of symptoms, until definitive therapy was implemented. Mediastinal calcification is commonly seen on CT imaging and not much clinical importance is attributed to it unless there is presence of symptomatic broncholithiasis. This case emphasizes the need to carefully review the degree of mediastinal calcification and comparison with prior imaging in cases presenting with mediastinal calcification and recurrent pulmonary infections.

\section{References}

1. Braimbridge MV, Keith HI. Oesophago-bronchial fistula in the adult. Thorax 1965;20:226-33.

2. Zhang BS, Zhou NK, Yu CH. Congenital bronchoesophageal fistula in adults. World J Surg 2011;17:1358-61.

3. Zhu J, Ni Y, Lu Q, Li X, Wang W, Wang H. Benign esophago-pulmonary fistula complicating achalasia: case report and literature review. J Thorac Dis 2015;7:E92-6.

4. Schreiber J, Waldburg N. Bronchoesophageal fistula and fatal hemoptysis after bevacizumab-containing chemotherapy without radiation in lung cancer. J Clin Oncol 2012;30:e324.

5. Park CS, Seo KW, Park CR, Nah YW, Suh JH. Case of bronchoesophageal fistula with gastric perforation due to multidrug-resistant tuberculosis. World J Gastrointest Surg 2014;6:253-8.

6. Lambertz R, Holscher AH, Bludau M, et al. Management of tracheoor bronchoesophageal fistula after ivor-lewis esophagectomy. World J Surg 2016;40:1680-7.

7. Camagni S, Lucianetti A, Ravelli P, et al. The successful management of a bronchoesophageal fistula after lung transplantation: a case report. Transpl Int 2015;28:884-7.

8. Faber LP, Jensik RJ, Chawla SK, Kittle CF. The surgical implication of broncholithiasis. J Thorac Cardiovasc Surg 1975;70:779-89.

9. Ford MA, Mueller PS, Morgenthaler TI. Bronchoesophageal fistula due to broncholithiasis: a case series. Resp Med 2005;99:830-5.

10. Dakkak M, Siddiqi F, Cury JD. Broncholithiasis presenting as bronchiectasis and recurrent pneumonias. BMJ Case Rep 2015; 2015:pii: bcr2014209035.

11. Ahn JY, Jung HY, Choi JY, et al. Benign bronchoesophageal fistula in adults: endoscopic closure as primary treatment. Gut Liver 2010; 4:508-13.

12. Deshpande G, Samarasam I, Banerjee S, et al. Benign esophagorespiratory fistula: a case series and a novel technique of definitive management. Dis Esophagus 2013;26:141-7.

13. Shen KR, Allen MS, Cassivi SD, et al. Surgical management of acquired nonmalignant tracheoesophageal and bronchoesophageal fistulae. Ann Thoracic Surg 2010;90:914-8; discussion 919. 